Ann. Génét. Sél. anim., I974, 6 (4), 405-414.

\title{
ÉTUDE GÉNÉTIQUE DE LA COLORATION BLEUE DE LA COQUILlE DES CEUFS CHEZ LA POULE : COMPARAISON DES PERFORMANCES DES ANIMAUX Oo ET oo
}

\author{
J. SILBER et P. MÉRAT \\ Laboratoire de Génétique factorielle, \\ Centre national de Recherches zootechniques, I. N.R. A., \\ 78350 Jouy en Josas
}

\section{RÉSUMÉ}

Des poules hétérozygotes pour le gène "œuf bleu " $(O o)$ ont un pourcentage d'œufs cassés en batteries de ponte réduit d'environ moitié par rapport à leurs sœurs pondant des oufs non bleus $(o o)$. Ceci paraît se retrouver à deux températures différentes. L'épaisseur moyenne des coquilles (membrane coquillière comprise) est sensiblement la même pour les deux génotypes, mais ces derniers présentent une différence significative pour la déformation élastique. Des observations annexes suggèrent la possibilité d'une plus grande épaisseur de la membrane coquillière interne pour les œufs des poules Oo. Les deux génotypes ne paraissent pas, d'autre part, présenter de différence pour les autres caractères d'intérêt économique. L'utilisation possible du gène $O$ dans certaines conditions est suggérée.

\section{INTRODUCTION}

A 1'heure actuelle on a mis en évidence un certain nombre d'associations entre des gènes connus et des caractères quantitatifs (voir par exemple revue par MÉRAT, I970) ; mais ce n'est que rarement qu'on a dressé, en vue de son utilisation en sélection, un tableau complet des effets quantitatifs associés aux différents génotypes à un locus. Le but de ce travail est de le faire pour le gène " œuf bleu ".

On trouve des poules pondant des œufs à coquille bleue appelées "Araucanas ", en Amérique Centrale et au Chili. Le déterminisme génétique de la ponte d'œufs de cette coloration a été mis en évidence par PunNETTT (I933). Ce dernier a montré qu'il s'agit d'un allèle autosomal dominant $(O)$, les poules oo pondant des œufs à coquille blanche ou brune.

Des poules "Araucanas ", introduites aux États-Unis et en Europe, ont été étudiées par différents auteurs pour le déterminisme de la pigmentation des coquilles (REUTER, I966) et pour leurs performances (BONADONNA, I970). 


\section{MATÉRIEL E'T MÉTHODES}

La population étudiée contenant divers gènes maintenus en ségrégation a été décrite par MÉRAT (1962). L'allèle $O$ y avait été introduit en 1965 à partir d'œufs à couver fournis par l'élevage de M. Hey of Heyfield, Turriff (Écosse).

Pour ce gène, la comparaison des moyennes des génotypes hétérozygote et homozygote récessif relative à un caractère est faite sur des couples de sœurs élevées ensemble. L'allèle $O$ est lié à $P$ (crête en pois) et $o$ à $p$ (crête simple). Il en est tenu compte dans l'interprétation des résultats. Au total, la comparaison des génotypes au locus $O$ comprend des données allant de 1967 à 1974 inclus.

Les animaux, éclos à l'automne et élevés au sol sont placés à l'âge de I 7 semaines en batterie de ponte. Les caractères suivants sont notés de 1967 à 1972 inclus : poids à 8 semaines; âge au $I^{\text {er }}$ œuf ; nombre d'œufs pondus jusqu'à l'âge de I I mois ; poids de l'animal adulte ; poids moyen des œufs sur 2 semaines à Io mois d'âge ; hauteur de l'albumen épais (mesure faite sur 2 cufs par poule à Io mois d'âge) ; épaisseur de la coquille à l'équateur de l'œuf, membrane coquillère comprise, chaque poule étant caractérisée par la moyenne de deux oufs consécutifs également à ro mois d'âge ; pourcentage d'œufs sans coquille (œufs "mous") et d'œufs "doubles "pondus jusqu'à I I mois. En outre, en I972, I973 et I974, le pourcentage d'œufs cassés en batterie est obtenu par des observations faites pendant I mois en début de ponte ; en 1973 et I974, la déformation élastique est estimée grâce à l'appareil décrit par Schoorl et BoERsma (I962) sur la partie équatoriale de l'œuf, mesure faite sur les œufs pondus durant une semaine pour chaque poule.

Dans une expérience séparée en 1974, des poules des génotypes Oo et oo ont été élevées dans un milieu ambiant maintenu à $34^{\circ} \mathrm{C}$ (lot chauffé) et comparées à un lot témoin (ambiance de $20^{\circ}$ ) sur une période de 3 mois après le début de la ponte. Nous avons comparé pour les poules des deux génotypes, le pourcentage d'œufs cassés sur une période de i I semaines, et d'autre part, sur une période d'une semaine : le nombre d'œufs à taches de sang; la hauteur d'albumen ; l'épaisseur de la coquille (dans la partie équatoriale, au voisinage du gros bout de l'œuf et au gros bout de l'œuf mais sans membrane interne) ; sa déformation élastique ; la perte de poids de l'œuf mis en incubateur pendant $4^{8}$ heures à $3^{\circ} \mathrm{C}$, ramenée à l'unité de surface, la surface de l'œuf étant estimée à partir de son poids selon la formule utilisée par TYLER et GEAKE (I960).

\section{RÉSULTATS}

\section{I. - Comparaison des performances des poules de génotype Oo et oo}

Le tableau I présente les valeurs moyennes des divers caractères quantitatifs pour les couples de sœurs $0 / 00$, calculées sur 6 années (r967 à I972 inclus).

\section{TABLEAU I}

Comparaison des performances moyennes des couples de sceurs de génotype Oo et oo (Moyenne de 6 années, 1967 à 1972 inclus)

\begin{tabular}{|c|c|c|c|c|c|}
\hline \multirow{2}{*}{ Caractère } & \multirow{2}{*}{$\begin{array}{c}\text { Nombre } \\
\text { de couples }\end{array}$} & \multicolumn{2}{|c|}{ Moyenne } & \multirow{2}{*}{$t$} & \multirow{2}{*}{ Signification } \\
\hline & & Oo & $o o$ & & \\
\hline Poids à 8 semaines $(\mathrm{g})$ & 86 & 724,8 & 708,2 & 1,58 & NS \\
\hline Age au $1^{\text {er }}$ œuf $(j) \ldots \ldots \ldots$ & 94 & 155,5 & 155,9 & 0,01 & NS \\
\hline Nombre d'cufs $\ldots \ldots \ldots \ldots$ & 94 & $92,2 ! z$ & 93,57 & 0,58 & NS \\
\hline Poids de l'œuf $(g) \ldots \ldots \ldots$ & 94 & $53,0^{\prime}{ }_{t}$ & 53,91 & 1,39 & NS \\
\hline Poids adulte $(g) \ldots \ldots \ldots \ldots$ & 92 & 1985,6 & 2062,\}^{\prime}$ & $2,46^{*}$ & $\mathrm{P}<0,05$ \\
\hline
\end{tabular}


Les différences sont peu importantes et non significatives, mis à part le fait que les poules adultes à œufs bleus sont un peu plus légères que leurs sœurs. Le linkage étroit existant entre les allèles $O$ et $P$ (crête en pois) permet difficilement à première vue de savoir lequel des deux allèles est en cause dans cette différence. Cependant l'absence de différence significative pcur le poids adulte entre poules à crête en pois et à crête simple indépendamment du gène $O$ (données non publiées) suggère que ce dernier peut être responsable des résultats présents.

\section{2. - Comparaison de caractéristiques de l'œuf}

a) Pourcentage d'oufs cassés en batterie.

Le tableau 2 donne les résultats des trois séries d'observations faites sur des troupeaux différents.

TABLEAU 2

Comparaison du pourcentage moyen d'aufs cassés pour des couples de saurs de génotype Oo et oo

\begin{tabular}{|c|c|c|c|c|c|}
\hline \multirow{2}{*}{ Année } & \multicolumn{2}{|c|}{ Pourcentage moyen } & \multirow{2}{*}{$\begin{array}{c}\text { Nombre } \\
\text { de couples }\end{array}$} & \multirow{2}{*}{$t$} & \multirow{2}{*}{ Signification } \\
\hline & Oo & $\infty 0$ & & & \\
\hline 72 & 6,25 & 13,43 & 56 & 2,06 & $P<0,05$ \\
\hline 73 & 8,03 & 13,88 & 46 & 2,24 & $\mathrm{P}<0,05$ \\
\hline 74 & 4,91 & 10,25 & 29 & 1,89 & NS \\
\hline Total & 6,62 & 12,88 & 131 & 3,56 & $\mathrm{P}<0,001$ \\
\hline
\end{tabular}

Dans les trois troupeaux l'écart du pourcentage d'œufs cassés entre les poules de génotype $O o$ et leurs sœurs $o o$ reste à peu près constant. Le résultat est significatif au seuil I p. I ooo.

b) Autres caractéristiques de l'œuf.

Les résultats sont rassemblés dans le tableau 3.

TABLEAU 3

Comparaison de caractéristiques de l'ceuf pour des couples de saurs de génotype Oo et oo

\begin{tabular}{|c|c|c|c|c|c|}
\hline \multirow{2}{*}{ Caractère } & \multirow{2}{*}{$\begin{array}{c}\text { Nombre } \\
\text { de couples }\end{array}$} & \multicolumn{2}{|c|}{ Moyenne } & \multirow{2}{*}{$t$} & \multirow{2}{*}{ Signification } \\
\hline & & Oo & oo & & \\
\hline Hauteur albumen $1 / 10 \mathrm{~mm})\left({ }^{1}\right) \ldots$ & 90 & 62,81 & 61,82 & 0,70 & NS \\
\hline Épaisseur coquille $(1 / 100 \mathrm{~mm})\left({ }^{1}\right)$. & 91 & 37,15 & 37,73 & 0,99 & NS \\
\hline CEufs mous $(\%)\left({ }^{2}\right) \ldots \ldots \ldots \ldots$ & 142 & 1,28 & 1,59 & 1,53 & NS \\
\hline Eufs doubles $(\%)(1973) \ldots \ldots \ldots$ & 46 & 0,75 & 0,26 & 1,30 & NS \\
\hline Déformation (1973 et 74$) \ldots \ldots$ & 56 & 22,10 & 23,89 & 2,89 & $P<0,01$ \\
\hline
\end{tabular}

(1) Moyenne de 6 années, 1967 à 1972 inclus.

$\left({ }^{2}\right)$ Inclus en plus des données de 1973 et 1974. 
J. SILBER, P. MÉRAT
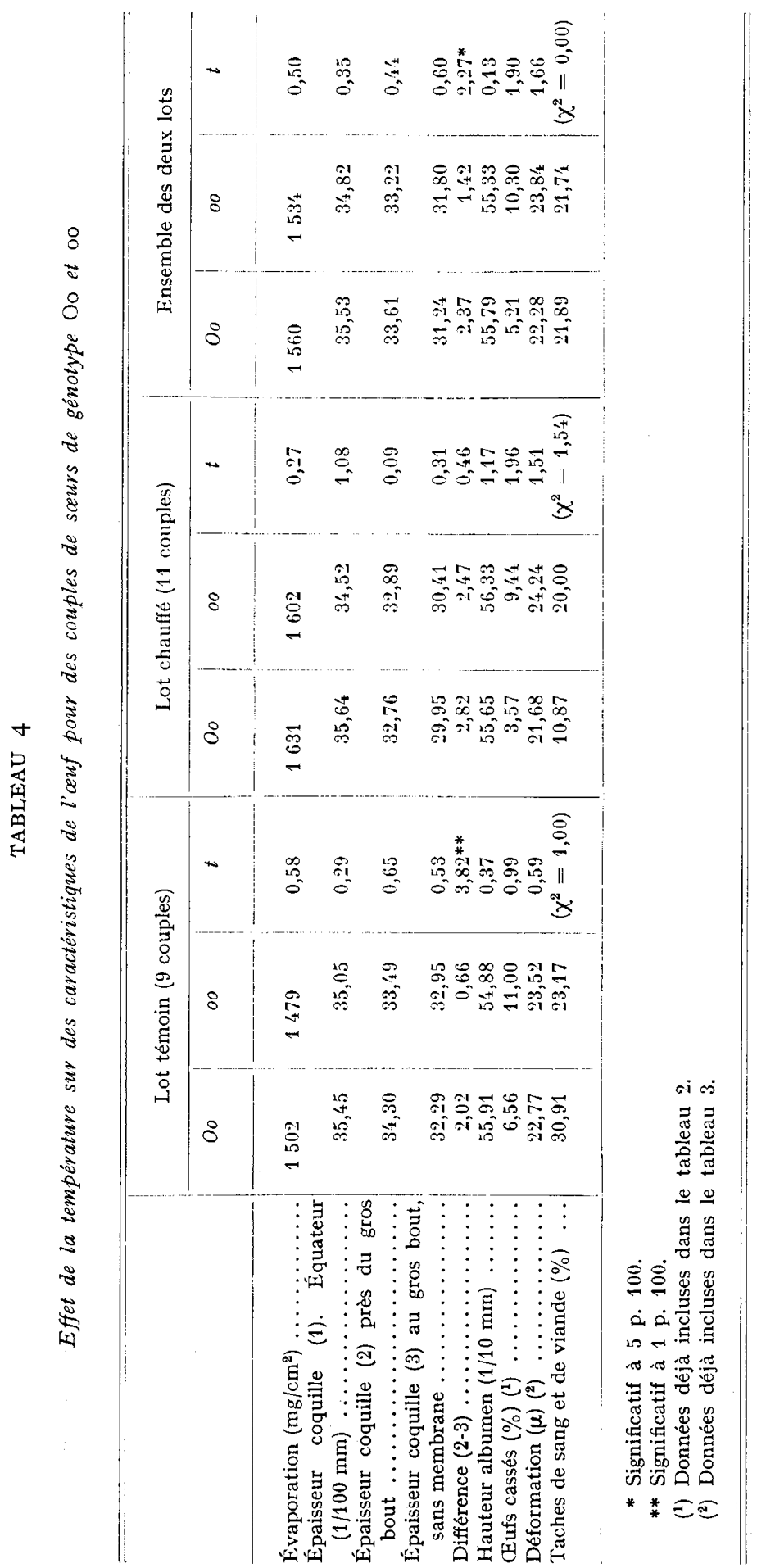
Nous observons une déformation de l'œuf significativement inférieure pour les poules $O o$ par rapport à leurs sœurs oo.

Il ne paraît pas y avoir de différences pour d'autres caractéristiques de l'œuf. Toutefois, celle concernant le pourcentage d'œufs "mous" (sans coquille) bien que faible, s'est répétée dans le même sens pour les 5 troupeaux étudiés.

3. - Comparaison des caractéristiques de l'œuf pour des poules de génotype Oo et oo à deux températures, et effet de la température

Les résultats sont donnés dans le tableau 4. Les différences ont d'abord été comparées intra-lot par la méthode des couples pour tous les caractères sauf pour les taches de sang. Pour celles-ci le nombre d'œufs par poule étant insuffisant pour calculer des pourcentages individuels nous avons testé les différences par un $\chi^{2}$ de contingence. Les mêmes tests ont été faits sur l'ensemble des deux lots " témoin " et " chauffé ", en l'absence d'effet significatif de la température sur tous les caractères considérés sauf un (cf. plus loin) et d'interaction décelable de ce facteur avec le génotype au locus $O$.

La perte de poids de l'œuf par évaporation ne diffère pas significativement pour les génotypes $O o$ et 00 ; elle est légèrement inférieure en valeur absolue pour les poules du lot chauffé. Toutefois, si l'on exprime cette perte de poids en fonction de la surface de l'œuf la différence s'annule (tab1. 4).

Il n'y a pas de différence dans l'épaisseur de la coquille entre les poules Oo et oo. Cependant, il existe un écart significatif entre ces poules pour la différence de mesure de 1'épaisseur de la coquille près du gros bout de l'œuf par rapport à celle faite au gros bout mais sans les membranes. La différence entre ces deux mesures comprend probablement une diminution réelle dans l'épaisseur de la coquille selon un axe longitudinal, mais surtout l'épaisseur de la membrane coquillère interne. Quant à l'effet de la température, en comparant globalement les lots témoin et chauffé, on peut dire que la chaleur paraît diminuer l'épaisseur de la coquille mais surtout dans le bout de l'œuf. En effet en cumulant les données des deux génotypes on trouve le résultat suivant (tabl, 5).

TABLEAU 5

Épaisseur en différents points de la coquille à deux températures différentes

\begin{tabular}{|c|c|c|c|}
\hline \multirow{2}{*}{$\begin{array}{l}\text { Localisation } \\
\text { de la mesure }\end{array}$} & \multicolumn{2}{|c|}{$\begin{array}{l}\text { Épaisseur de la coquille } \\
(1 / 100 \mathrm{~mm})\end{array}$} & \multirow{2}{*}{$t$} \\
\hline & Lot témoin & Lot chauffé & \\
\hline Équateur ......... & 35,61 & 35,25 & 0,51 \\
\hline Près du gros bout ...... & 33,96 & 33,44 & 0,72 \\
\hline Gros bout sans membrane & 2,521 & $30,7 !$ & 2,28 * \\
\hline
\end{tabular}

d.1. $=57$.

* Significatif à 5 p. 100. 
Pour l'épaisseur de l'albumen, l'absence d'effet significatif associé au gène $O$ est conforme aux résultats précédents obtenus sur des effectifs plus importants (cf. tab1. 3). La différence entre génotypes $O o$ et oo pour le pourcentage d'œufs cassés et la déformation élastique, quoique non significative ici, va dans le même sens que les résultats des tableaux 2 et 3 . Enfin, le tableau 4 ne suggère aucun effet attribuable au gène $O$ sur le pourcentage de taches de sang.

Nous observons d'autre part, en ce qui concerne l'effet de la chaleur, une légère augmentation de l'épaisseur de l'albumen proche du seuil $5 \mathrm{p}$. Ioo de signification : 55,67 pour le lot témoin, 58,47 pour le lot chauffé, $t=\mathrm{r}, 93$ pour 57 d.1. Par contre, il n'y a pas d'effet de la température sur le pourcentage d'œufs cassés, ni sur le pourcentage de taches de sang, ni sur la déformation de l'œuf.

\section{4. - Corrélations entre diverses caractéristiques de l'œuf à deux températures}

Les diverses corrélations sont données dans le tableau 6 pour le lot témoin et dans le tableau 7 pour le lot chauffé, les génotypes $O o$ et 00 étant groupés.

TABLEAU 6

Corrélations entre diverses caractéristiques de l'auf

(Lot témoin)

\begin{tabular}{|c|c|c|c|c|c|c|}
\hline & Déformation & $\begin{array}{l}\text { Hauteur } \\
\text { albumen }\end{array}$ & $\begin{array}{l}\text { Épaisseur } \\
\text { coquille } \\
\text { à l'équateur }\end{array}$ & $\begin{array}{l}\text { Épaisseur } \\
\text { coquille près } \\
\text { du gros bout }\end{array}$ & $\begin{array}{c}\text { Épaisseur } \\
\text { coquille au } \\
\text { gros bout sans } \\
\text { membrane }\end{array}$ & $\begin{array}{c}\text { Perte de poids } \\
\text { par } \\
\text { évaporation }\end{array}$ \\
\hline CEufs cassés (\%) & $+0,585^{* * *}$ & $-0,102$ & $-0,499^{* *}$ & 一 $0,402 *$ & $-0,282$ & $-0,176$ \\
\hline Déformation & 1 & $+\theta, 078$ & $-0,700^{* * *}$ & $-0,524 * *$ & 一 $0,410^{*}$ & $+0,353$ \\
\hline $\begin{array}{l}\text { Hauteur } \\
\text { albumen }\end{array}$ & & 1 & $+0,292$ & $+0,196$ & $+0,090$ & $+0,453^{*}$ \\
\hline $\begin{array}{l}\text { Épaisseur } \\
\text { coquille } \\
\text { équateur }\end{array}$ & & & 1 & $+0,886^{* * *}$ & $+0,720^{* * *}$ & $+0,015$ \\
\hline $\begin{array}{l}\text { Épaisseur } \\
\text { coquille près } \\
\text { gros bout }\end{array}$ & & & & 1 & $+0,885^{* * *}$ & \\
\hline
\end{tabular}

d.1. $=29$.

* Significatif à 5 p. 100.

** Significatif à 1 p. 100.

*** Significatif à 1 p. 1000.

Il y a une liaison hautement significative entre le pourcentage d'œufs cassés et la déformation dans les deux lots, ainsi qu'avec la hauteur du blanc épais dans le lot chauffé. 
La liaison entre le pourcentage d'œufs cassés et les diverses mesures de l'épaisseur de la coquille est toujours négative, mais n'est hautement significative qu'avec la mesure faite à l'équateur de l'œuf et seulement dans le lot témoin.

TABLEAU 7

Corrélations entre diverses caractéristiques de l'ceuf

(Lot chauffé)

\begin{tabular}{|c|c|c|c|c|c|c|}
\hline & Déformation & $\begin{array}{l}\text { Hauteur } \\
\text { albumen }\end{array}$ & $\begin{array}{l}\text { Épaisseur } \\
\text { coquille } \\
\text { à l'équateur }\end{array}$ & $\begin{array}{c}\text { Épaisseur } \\
\text { coquille près } \\
\text { du gros bout }\end{array}$ & $\begin{array}{c}\text { Épaisseur } \\
\text { coquille au } \\
\text { gros bout sans } \\
\text { membrane }\end{array}$ & $\begin{array}{l}\text { Perte de poids } \\
\text { par } \\
\text { évaporation }\end{array}$ \\
\hline CEufs cassés $(\%)$ & $+0,534 * *$ & $+0,511^{* *}$ & $-0,237$ & $+0,061$ & $-0,233$ & $+0,024$ \\
\hline Déformation & 1 & $+0,425 *$ & $-0,788^{* * *}$ & $-0,635^{* * *}$ & $-0,716^{* * *}$ & $+0,165$ \\
\hline $\begin{array}{l}\text { Hauteur } \\
\text { albumen }\end{array}$ & & 1 & $-0,308$ & $-0,152$ & $-0,231$ & $-0,019$ \\
\hline $\begin{array}{l}\text { Épaisseur } \\
\text { coquille } \\
\text { équateur }\end{array}$ & & & 1 & $+0,767 * * *$ & $+0,672^{* * *}$ & $-0,077$ \\
\hline $\begin{array}{l}\text { Épaisseur } \\
\text { coquille près } \\
\text { gros bout }\end{array}$ & & & & 1 & $+0,866^{* * *}$ & \\
\hline $\begin{array}{r}\text { d.l. }=26 . \\
* \text { Signific } \\
* * \text { Signific } \\
* * * \text { Signific }\end{array}$ & $\begin{array}{l}\text { atif à } 5 \text { p. } 100 . \\
\text { atif à } 1 \text { p. } 100 . \\
\text { atif à } 1 \text { p. } 1000\end{array}$ & & & & & \\
\hline
\end{tabular}

L_es différentes mesures faites sur l'épaisseur de coquille en différents endroits de l'œuf sont fortement liées entre elles.

Les liaisons entre la hauteur du blanc épais et les différents caractères paraissent varier considérablement pour les deux lots. Les coefficients de corrélation de cette mesure avec le pourcentage d'œufs cassés ainsi qu'avec l'épaisseur de la coquille à l'équateur sont statistiquement différents aux deux températures $(P<0,025$ dans les deux cas).

La perte de poids par évaporation n'est liée qu'avec la hauteur d'albumen et seulement dans le lot témoin ; toutefois, sa corrélation avec la déformation va dans le même sens pour les deux lots.

\section{DISCUSSION - CONCLUSIONS}

Le résultat le plus intéressant de ce travail nous paraît être la différence observée dans le pourcentage d'œufs cassés en batterie entre les poules de génotype $O o$ et celles 
de génotype $o o$ : L'avantage lié aux animaux de génotype $O o$ paraît être répétitif et la différence est très hautement significative dans l'ensemble.

On pourrait tenter d'expliquer en partie ce résultat par le fait que les poules à œufs bleus sont significativement plus légères que leurs sœurs à œufs non bleus, car le poids de l'animal est en corrélation positive avec le pourcentage d'œufs cassés en batterie (SIIBER, I974).

Cependant, ceci ne pourrait expliquer l'écart observé que dans une faible mesure : d'après la régression de la deuxième variable sur la première, on ne pourrait estimer qu'à environ 0,2 p. Ioo la diminution du pourcentage des œufs cassés causée par la réduction du poids corporel de $77 \mathrm{~g}$ associée au génotype $O o$.

On peut émettre d'autres hypothèses : celle d'une différence de structure de la coquille, suggérée par la différence significative observée dans la déformation de l'œuf sous un poids donné (CARTER, I969) ; ou encore celle d'une différence dans l'épaisseur des membranes coquillères entre les poules de génotype $O o$ et celles de génotype oo. En effet, on a vu dans le tableau 4 que la différence des mesures 2 et 3 est significativement supérieure pour les poules $O o$, or cette mesure comprend l'épaisseur des membranes coquillères.

Le pourcentage d'œufs mous est légèrement plus faible pour les poules $O_{o}$; il est probable que cela est dû en partie aux mêmes raisons que pour les œufs cassés, la liaison entre les deux caractères étant significative (LACASSAGNE et MoNGIN, I965; SIIBER, I974).

En ce qui concerne les observations annexes, indépendantes du génotype au locus $O$, nos résultats sur la diminution de l'épaisseur de la coquille selon un axe longitudinal sont à rapprocher de ceux de TYLER (I96I) : sur 23I œufs cassés la moyenne de l'épaisseur de la coquille à l'équateur est de $35,7 \mathrm{I}$, celle près du gros bout de 33,9I et la différence par un test des couples est significative à I p. Iooo $(t=\mathrm{I} 4,03)$.

Par contre, l'effet de la température sur l'épaisseur des coquilles (au gros bout de l'œuf) est conforme aux résultats trouvés par d'autres auteurs (WARREN et SCHNEPEL, I940), ainsi que les corrélations observées entre mesures de déformation et d'épaisseur de coquille (Brooks et HALE, I955).

Le problème se pose de savoir si une utilisation pratique peut être tirée des résultats présentés ci-dessus.

La casse des œufs est une perte importante pour 1'aviculture et il apparaît dans notre population, et pour le type de batterie utilisé par nous, une diminution d'à peu près 6,5 p. Ioo dans le pourcentage d'œufs cassés par poule pondant des oufs à coquille bleue comparée à une poule oo d'origine identique.

Les poules de ces deux génotypes ne paraissent pas, d'autre part, présenter de différence pour les autres caractères économiques dont on tient compte en sélection avicole.

On ne peut encore établir avec certitude, à l'heure actuelle, si cette diminution du pourcentage d'œufs cassés est un effet pléiotropique du gène $O$ ou provient d'un étroit linkage. Cependant, le fait même que l'effet visible du gène $O$ concerne précisément une caractéristique de la coquille et des membranes coquillères paraît plutôt favorable, a priori, à la première hypothèse. D'autre part, des données relatives au gène $P$ en dehors de la présence du gène $O$, n'indiquent aucun effet associé à ce gène sur le pourcentage d'œufs cassés (SILBER et MÉRAT, I974). Un moyen complémen- 
taire de tester l'hypothèse d'un effet pléiotropique devrait être l'étude de la proportion d'œufs cassés et des caractéristiques de leurs coquilles dans différentes populations en ségrégation pour les allèles $O$ et $o$.

Si l'amplitude des différences dans le pourcentage d'œufs cassés et leur origine pléiotropique se confirment, l'introduction de l'allèle $O$ dans des souches mères de poulets de chair pourrait être intéressante surtout au cas où dans l'avenir les reproductrices de ces souches seraient en batterie. On peut également envisager son utilisation lorsque les œufs servent uniquement à un usage industriel et même éventuellement dans des souches de ponte, dans des cas où la coloration de la coquille ne serait pas un obstacle pour le consommateur.

Reçu pour publication en septembre 1974.

\section{REMERCIEMEN'TS}

Nous remercions M. P. Mongin (Station de Recherches avicoles, I. N. R. A., 37 Nouzilly) et M. Legault (Station de Génétique quantitative et appliquée, I. N. R. A., 78 Jouy en Josas) de leurs remarques et critiques constructives concernant ce manuscrit.

Nous sommes également reconnaissants à MM. Hey of Heyfield, TurRiff (Écosse) de nous avoir fourni les œufs à couver à partir desquels le gène "œuf bleu » a été introduit dans notre troupeau.

\section{SUMMARY}

GENETIC STUDY OF THE BLUE EGG SHELL COLOUR IN THE FOWL : COMPARISON OF PERFORMANCE OF OO AND OO BIRDS

Hens heterozygous for the "blue egg " gene $(O o)$ give a percentage of broken eggs in laying batteries reduced about by half, as compared to their oo full-sisters. This seems to be the same at a high ambient temperature. The average shell thickness (including shell membrane) is about the same for the two genotypes, but there is a significant difference for elastic deformation. Other observations suggest the possibility for the inner shell membrane to be thicker for eggs from $O o$ hens.

On the other hand, the two genotypes do not present any other significant difference for traits of economic value. Possible use of the $O$ gene in certain conditions is suggested.

\section{RÉFÉRENCES BIBLIOGRAPHIQUES}

Bonadonna T., 1970. La gallina Araucana dalle uova azzure. Zootec. Vet., 25, r30-I32.

Brooks J., Hale H. P., 1955. Strength of the shell of the hen's egg. Nature, Lond., 175, 848-849.

CARTER T. C., rg69. The hen's egg : Variation in shell deformation under static load with relative humidity and age of egg. Br. Poult. Sci, 10, 3I I-319.

Knox C. W., Godfrey A. B., I934. Variability of thick albumen in fresh-laid eggs. Poult. Sci., 13, I8-22.

Lacassagne L., Mongin P., I965. Maturité sexuelle et qualité de la coquille de l'œuf. Ann. Zootech., 14, 327-334.

MÉrat P., r962. Quelques relations entre caractères extérieurs à hérédité simple et productivité. Proc. $X I I$ th. World's Poultry Congress, Sydney, Section papers, $71-76$. 
Mérat P., I97o. Mendelian genetics and selection for quantitative traits in Poultry : Results and perspectives. World's Poult. Sci. J., 26, 57I-586.

Punnetr R. C., I933. Genetic studies in Poultry. IX. The blue egg. J. Genetics, 27, 465-470.

Reuter W., rg66. Studien über blaue Vogeleier. Zeit. Wissensch. Zool., 173, 245-338.

Schoorl P., Boersma H. Y., I962. Research on the quality of the egg shell. A new method of determination. I2 th. Wld's Poult. Congr., Sydney, 432-435.

Silber J., 1974. Etude génétique de caractéristiques de l'œuf de Poule. Thèse $3^{\circ}$ cycle. Université Paris VI.

Silber J., Mérat P., 1974. Étude génétique des œufs cassés en batterie de ponte. Proc. XVth World's Poult. Congress, New Orleans, 266-268.

TYLER C., r96r. Studies on egg shells. XVI. Variation in shell thickness over different parts of the same shell. J. Sci., Fd. Agric., 12, 459-475.

Tyler C., Geake H. F., r96o. Studies on egg shells. XIII. Influence of individuality, breed, season and age on certain characteristics of egg shells. J. Sci. Fd. A gric., 11, 535-547.

Warren D. C., Schnepel R. C., r940. The effect of air temperature on egg shell thickness in the fowl. Poult. Sci., 19, 67-72. 